\title{
Responsabilidad social empresarial en las franquicias farmacéuticas*
}

\author{
Brooks, Nicolás** \\ Pérez Frías, Arturo*** \\ Pérez R., Ingrid E.****
}

\section{Resumen}

Atender la problemática social y lograr la calidad de vida de los individuos es obligación del Estado, pero es también un deber de los empresarios. En tal sentido, el propósito del presente artículo es identificar la presencia de la responsabilidad social empresarial en los elementos estratégicos: misión, visión, valores, políticas y normas. Se hizo un estudio exploratorio, de enfoque cuantitativo, no experimental, transeccional y de campo, sobre las franquicias farmacéuticas en actividad en el Municipio Maracaibo en el 2006. Los resultados señalan la presencia de la responsabilidad social empresarial (RSE) en los elementos estratégicos de ambas franquicias. Se concluye que reconocer esa presencia resalta la necesidad de profundizar en la aplicación del concepto de RSE sobre la gestión para afianzarla más, mejorando su asimilación, fortaleciendo acciones en cumplimiento de la obligación ética y moral asumida voluntariamente por estas empresas y midiendo el impacto generado de acuerdo a los objetivos escogidos dentro del marco de la RSE, en función de mejorar calidad de vida y satisfacción de demandas de orden social a nivel interno y externo de dichas organizaciones. Además, mantener y superar el comportamiento responsable con la sociedad, representa un factor de competitividad de primer orden por las exigentes expectativas de las partes involucradas o grupos de interés, pues no hacerlo traería perdida de mercado, desconfianza y rechazo, aspectos contrarios al éxito y permanencia en el tiempo de estas empresas.

Palabras clave: Responsabilidad social empresarial, ética, moral, franquicias farmacéuticas, elementos estratégicos.

\section{Recibido: 21-11-07 Aceptado: 12-01-09}

* Este artículo se basa en los resultados obtenidos en la investigación "Responsabilidad Social en las Franquicias Farmacéuticas del Municipio Maracaibo", dependiente de la División de Investigación y Postgrado de la Universidad Dr. Rafael Belloso Chacín.

** Investigador. División de Investigación y Postgrado de la Universidad Dr. Rafael Belloso Chacín. Maracaibo, Estado Zulia, Venezuela.

*** Profesor-Investigador. Instituto de Investigaciones. Facultad de Ciencias Económicas y Sociales. Universidad del Zulia. Maracaibo, Estado Zulia, Venezuela. E-mail: aperezfrias@gmail. com (autor para correspondencia).

**** Profesora-Investigadora. Instituto de Investigaciones. Facultad de Ciencias Económicas y Sociales. Universidad del Zulia.Maracaibo, Estado Zulia, Venezuela. 


\title{
Corporate Social Responsibility in Drug Franchises
}

\begin{abstract}
Address social problems and achieving quality of life of individuals, it is the duty of the state, but is alsAddressing social problems and achieving a quality of life for individuals is the duty of the State, but it is also a duty of entrepreneurs. The purpose of this paper is to identify the presence of corporate social responsibility in the strategic elements: mission, vision, values, policies and standards. An exploratory study was made using a quantitative, non-experimental, trans-sectional and field approach, on active pharmaceutical franchise businesses in the Maracaibo Municipality in 2006, using a census with the media managers for the two companies. Results suggest the presence of corporate social responsibility (CSR) in the strategic elements of both franchises. Conclusions are that recognizing this presence highlights the need to deepen application of the CSR concept on management to make it more secure, improve its assimilation, reinforce actions that comply with the ethical and moral obligation voluntarily assumed by these companies, and measure the impact generated according to objectives chosen within the CSR framework in terms of improving quality of life and satisfying social demands at both the internal and external levels of these organizations. Also, maintaining and improving responsible behavior toward society represents a competitiveness factor of the first order for the demanding expectations of the involved parties or interest groups, because not demonstrating responsible behavior would bring a loss of market, lack of confidence and rejection, aspects contrary to the success and permanence of these companies over time.
\end{abstract}

Key words: Corporate social responsibility, ethics, moral, pharmaceutical franchises, strategic elements.

\section{Introducción}

La responsabilidad social empresarial (RSE) es un tema de actualidad, que ha generado gran interés en procura de conocer sus alcances y características. En tal sentido, Bull (2004) señala la existencia de grupos dentro de los cuales se incluyen la comunidad empresarial, instituciones internacionales, agencias de desarrollo, y movimientos activistas, que han adoptado la RSE y la ven como un camino para mejorar la calidad de vida de las personas y proteger el medio ambiente.

Pero muchas empresas en el ámbito supranacional (Álvarez, 2007), han tenido un comportamiento inapropiado o in- justo que abarca acciones irresponsables contra el medio ambiente, empleo de mano de obra infantil, corrupción, discriminación, publicidad engañosa, salarios mínimos por debajo del mínimo legal establecido, entre otras muchas prácticas inadecuadas y egoístas.

Estas situaciones que se perciben como contradictorias, sirven de base para comprender por qué Carneiro (2004), refiriéndose a la responsabilidad social, consideró que las empresas debían tener plena conciencia de los efectos reales que producen sus acciones sobre el entorno social, estableciendo formas de comportamiento orientadas hacia una actitud positiva en lo relacionado con los valores e intereses de la sociedad, lo 
cual subraya la pertinencia para los empresarios de dar respuestas positivas a las demandas de orden social, tanto por el peso específico de las empresas en el conjunto social como a la percepción que en el mismo tienen las cuestiones relacionadas con el bienestar y la calidad de vida.

De lo anterior se derivan dos aspectos: El primero, referido a la responsabilidad social entendida como obligación ética y moral, asumida voluntariamente por la empresa, por formar parte del marco social. El segundo, relativo al contenido operativo del concepto de la responsabilidad social, lo cual significa reunir un conjunto de indicadores ordenados que permitan llevar a cabo una clasificación de las incidencias sociales, la participación de las organizaciones y la medición del impacto que el cumplimiento de la obligación ética logra para mejorar las condiciones y satisfacer las demandas de orden social de las comunidades a las cuales dichas organizaciones hayan escogido voluntariamente en el cumplimiento de los objetivos establecidos dentro del marco de la responsabilidad social.

Considerando la RSE como obligación ética, Carneiro (2004) señala que las empresas deben asumir tres niveles de compromiso:

1. Las responsabilidades primarias inmediatas de la empresa, las cuales la vinculan con los integrantes de la propia organización (accionistas, directivos y trabajadores).

2. Las responsabilidades secundarias, orientadas hacia los agentes del entorno específico de la empresa (proveedores de insumos, servicios, productos, financistas, distribuidores, clientes, consumidores y competidores).

3. Las responsabilidades frente al entorno general de la organización, en especial el Estado y la administración pública, así como las comunidades con las cuales se tenga relación.

Es decir, la responsabilidad social empresarial abarca entonces la responsabilidad económica, la sociocultural y la medioambiental. Frente a ese marco, señala Carneiro (2004) que empresas de renombre a nivel mundial han dedicado porciones importantes de sus recursos para invertirlos en programas e iniciativas, buscando incrementar la calidad de vida de los individuos en la sociedad dentro de la cual operan.

De esa forma, muchas organizaciones han logrado ampliar su razón de ser, entendiéndose no solamente como sociedades cuyos propósitos son producir dividendos para los accionistas, proporcionar empleo y generar ingresos fiscales, sino que adicionalmente, se han venido ocupando de asumir una conducta social suficientemente responsable, debido a que la empresa entendida como un ente con personalidad propia, inmersa en un ámbito social en el cual hace vida, debe proyectarse y entender que es necesario e imperativo garantizar calidad de vida al entorno que la rodea.

Al asumir las empresas voluntariamente tal compromiso colaborando con el desarrollo social, los derechos humanos y el cuidado medioambiental, aceptan trabajar por el logro de una mejor calidad de vida del entorno social, lo cual favorece el terreno para un trabajo en conjunto entre las empresas, pudiendo incorporarse de esta forma a un efectivo cum- 
plimiento de sus responsabilidades con la sociedad en la cual participan como ciudadanos corporativos.

Referirse a la responsabilidad social significa entonces que las empresas deciden de forma voluntaria en contribuir con una sociedad mejor y un medioambiente más limpio (Libro verde COM, 2001), asumiendo esto como parte de la práctica normal en la actividad empresarial, sobre la cual se hace necesario destacar la importancia que reviste el uso de formas para medir la RSE, haciendo referencia al conjunto de prácticas que definen el paradigma de la gestión empresarial responsable, tal como destaca Álvarez (2007), tomando en primera opción la integración de la RSE en la misión, visión, valores, políticas y normas existentes en la organización, luego la gestión de los grupos de interés, siguiendo con la gestión técnico-económica, gestión del gobierno corporativo, gestión social y gestión ambiental, en concordancia con lo destacado al principio del artículo, sobre la medición del impacto en el cumplimiento de la obligación ética.

A la vanguardia de una actitud responsable se destacan empresas norteamericanas y europeas. En América Latina también ha tenido eco en las empresas el reto de asumir una actitud responsable ante la sociedad. Sin embargo, dentro de los países latinos donde se percibe mejor actitud hacia la RSE es en Brasil y Chile; en otros países de la región se están haciendo importantes esfuerzos, pero las prácticas no se han extendido suficientemente y debe realizarse un trabajo intenso para lograr que los empresarios capten la importancia de contribuir a mejorar el medioambiente y la calidad de vida de las comunidades,

Con relación a este aspecto, Thompson (2000: 46) Director del programa para América Latina y Caribe de la Fundación Kellogg, acota lo siguiente:

En otros países, como Colombia, Argentina, México o Perú, a pesar de esfuerzos constantes de centros o núcleos de promoción, aún estas prácticas no consiguen extenderse y tener visibilidad. Aunque algunas fundaciones internacionales $u$ organismos multilaterales han apoyado esfuerzos en esa dirección, los líderes empresariales aún no logran entender a fondo el mensaje más básico y elemental que este movimiento quiere transmitir: el éxito de las empresas depende más que de ninguna otra cosa de la generación de un entorno favorable para su desarrollo.

Pero coincidiendo con Vives, $A$. et al. (2005), lo cierto es que el tema de la responsabilidad social empresarial ha tomado un interés creciente en el ámbito latinoamericano, tanto en el sector académico como en las empresas, especialmente en las grandes, colocando a la responsabilidad social como factor de importancia para el sector empresarial. Así, en 2003, autores como González I. A. et al. (citado en Vives 2005) sintetizan la percepción actual del concepto, al señalar que la responsabilidad social empresarial ha llegado para quedarse, de forma que visualizar la empresa solamente como productora de rentabilidad para sus dueños, pierde vigencia ante la realidad económica y social latinoamericana. 
En lo que respecta a Venezuela y de acuerdo con Méndez (2003), en la realidad nacional se aprecian esfuerzos orientados a la aplicación de prácticas en responsabilidad social, con orientaciones parecidas a las observadas en el resto de América Latina; diferentes grupos empresariales del país han desarrollado programas de RSE en su actuación frente al entorno social que les rodea.

En tal sentido, la intención del presente trabajo es detenerse en la realidad específica de las franquicias farmacéuticas cuyo origen geográfico es el municipio Maracaibo, a fin de identificar en ellas la presencia de la responsabilidad social en sus elementos estratégicos misión, visión, políticas y normas.

Para lograrlo, se realizó una investigación exploratoria, con un enfoque cualitativo en la revisión de los aspectos relacionados con la presencia de la RSE en los elementos estratégicos de las franquicias farmacéuticas del municipio Maracaibo, de acuerdo a los conceptos metodológicos de Hernández, et al. (2003), utilizando un diseño no experimental, transeccional y de campo.

En relación con la población, la Asociación Venezolana de Franquicias (2005), indicó que las franquicias farmacéuticas con actividad en el municipio Maracaibo del Estado Zulia, eran dos, Io cual llevó a considerar de acuerdo con el concepto de Parra (2000), que la población estaba compuesta por el conjunto de características y atributos percibidos por todos los gerentes medios de ambas empresas, en total 20 sujetos informantes responsables de cumplir y hacer cumplir lo establecido en los elementos estratégicos.
El tratamiento estadístico utilizado para la investigación fue de tipo descriptivo, de acuerdo con Chávez (2000).

\section{Presencia de la RSE en los elementos estratégicos}

Se identificó la presencia de RSE en los elementos estratégicos de las franquicias estudiadas, comparando esos resultados con la teoría en la cual se sustentó la investigación.

\subsection{Misión}

De acuerdo con Morrisey (2002), la declaración de la misión se plantea con el objeto de proporcionar una firme guía en la toma de decisiones administrativas impactantes, para establecer y mantener una consistencia y claridad de propósitos en toda la organización, así como proporcionar un marco referencial a todas las decisiones de planificación que se han de tomar en la junta directiva, su equipo administrativo y en las otras unidades departamentales de la organización, logrando el compromiso de todos los miembros de la organización mediante una comunicación clara de la naturaleza y el concepto del negocio, atrayendo la comprensión y el apoyo de personas externas que sean importantes para el éxito de la organización.

Por otra parte y según el Consejo Empresarial Mundial para el Desarrollo Sostenible (2002), la responsabilidad social empresarial es un compromiso de actuar éticamente, y de contribuir al crecimiento económico en procura de mejorar la calidad de vida de la fuerza laboral así como de sus familias. De esa forma, la 
responsabilidad social vista como obligación ética, conlleva a que las empresas deban asumir tres niveles de compromiso tal y como lo destaca Carneiro (2004): el económico, el sociocultural y el medioambiental.

Con base en los conceptos anteriores, se hizo evidente la necesidad de identificar en la misión la presencia del compromiso de los integrantes de ambas organizaciones a nivel externo e interno, en el marco de la responsabilidad social.

De acuerdo a los resultados reflejados en la Tabla 1 y el Gráfico 1, puede apreciarse como el $86.25 \%$ de los gerentes entrevistados coincidieron en señalar que siempre o casi siempre, en estas empresas se logra el compromiso de sus miembros con la responsabilidad social, por medio de una comunicación clara del concepto, así como consistencia del compromiso ético de la organización con sus relacionados del negocio farmacéutico.

El $13.75 \%$ restante prefirió las opciones "algunas veces" o "casi nunca", considerando que en estas organizaciones es baja la obtención del compromiso de los miembros para asumir un comportamiento ético y socialmente responsable a nivel externo e interno.

Los resultados anteriores muestran una tendencia favorable en función de la identificación de la misión como ele-

\section{Gráfico 1 \\ Presencia de RSE en la misión}

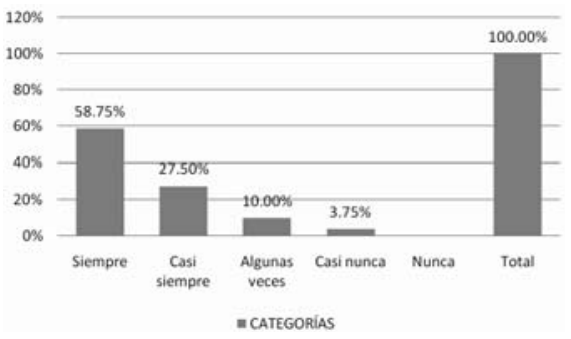

Fuente: Elaboración propia (2006).

mento estratégico orientado hacia la responsabilidad social en ambas franquicias, pues desde la óptica de los gerentes entrevistados estas empresas logran la mayoría de las veces el compromiso de los empleados, a través de una comunicación clara del concepto de misión hacia el entorno social y consistencia en el compromiso ético con sus relacionados del negocio farmacéutico, coincidiendo con lo expresado por Morrisey (2002).

\subsection{Visión}

El significado de una empresa socialmente responsable, implica algo más que cumplir con las obligaciones establecidas en las normativas jurídicas vigentes, tal como lo estipula la Comisión de las Comunidades Europeas (Libro Verde COM, 2001), pues se trata de invertir mu-

\section{Tabla 1}

Presencia de RSE en la misión

\begin{tabular}{lccccccc}
\hline & Categorías & Siempre & $\begin{array}{c}\text { Casi } \\
\text { siempre }\end{array}$ & $\begin{array}{c}\text { Algunas } \\
\text { veces }\end{array}$ & $\begin{array}{c}\text { Casi } \\
\text { nunca }\end{array}$ & $\begin{array}{c}\text { Nunca } \\
\text { Indicador: }\end{array}$ & $\begin{array}{c}N^{\circ} \text { de } \\
\text { Sujetos }\end{array}$ \\
\cline { 2 - 7 } Compromiso & F. Absoluta & 11,75 & 5,5 & 2 & 0,75 & 0 & 20 \\
& $\%$ & $58,75 \%$ & $27,5 \%$ & $10 \%$ & $3,75 \%$ & - & $100 \%$ \\
\hline
\end{tabular}

Fuente: Elaboración propia (2006). 
cho más en el capital humano y en el entorno social. Lo anterior se complementa con la sugerencia de que aquellas empresas que invierten en tecnología y en procesos respetuosos del ambiente por lo general ganan en competitividad a partir de la aplicación de normas más estrictas que las socialmente establecidas, lo cual también puede incidir en un aumento de la productividad y por ende de la competitividad.

Por otro lado, para Morrisey (2002) la visión es una representación de lo que los gerentes creen que el futuro debe ser para su empresa, a los ojos de los clientes, empleados, propietarios y otros accionistas importantes; su origen es casi por completo intuitivo, es una consecuencia de valores y convicciones del equipo de administración, para lo cual se debe establecer una planificación y coordinación con los objetivos de la organización (Gráfico 2).

A partir de los conceptos de responsabilidad social empresarial expresados por la Comisión (Libro Verde COM, 2001) y lo señalado por Morrisey (2002), se buscó identificar la presencia de la RSE en la Visión de cada una de las empresas consideradas, mediante la comprobación de la existencia de metas proyectadas en el mercado, sobre productos y servicios.

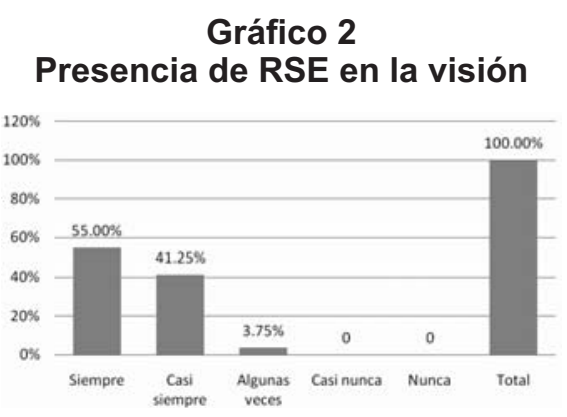

Fuente: Elaboración propia (2006).

Tal como lo refleja la Tabla 2, se encontró que de la totalidad de respuestas de los sujetos informantes, el $96,25 \%$ reflejó que la organización "siempre" o "casi siempre" proyectaba metas en el mercado al cual dirige su actividad productiva y en los productos que ofrecen y servicios que prestan, bajo el marco de la RSE, mientras un $3,75 \%$ señalaba la opción "algunas veces".

De esa forma se vio reflejada una situación positiva, pues los sujetos informantes, al considerar mayoritariamente que estas organizaciones tienden a proyectar sus metas y mostrar lo que quieren llegar a ser en el mercado dentro de un marco de responsabilidad social, están adecuándose a lo que los gerentes creen que el futuro deba ser para estas organizaciones, coincidiendo con lo destacado por Morrisey (2002).

\section{Tabla 2}

Presencia de RSE en la visión

\begin{tabular}{lccccccc}
\hline $\begin{array}{l}\text { Indicador: } \\
\text { Metas }\end{array}$ & Categorías & Siempre & $\begin{array}{c}\text { Casi } \\
\text { siempre }\end{array}$ & $\begin{array}{c}\text { Algunas } \\
\text { veces }\end{array}$ & Casi nunca & $\begin{array}{c}\text { Nunca } \\
\text { proyectadas }\end{array}$ & $\begin{array}{c}N^{\circ} \text { de } \\
\text { Sujetos }\end{array}$ \\
\cline { 2 - 8 } en mercado/ & F. Absoluta & 11 & 8,25 & 0,75 & 0 & 0 & 20 \\
$\begin{array}{l}\text { productos } \\
\text { lservicios }\end{array}$ & $\%$ & $55 \%$ & $41,25 \%$ & $3,75 \%$ & - & - & $100 \%$ \\
\hline
\end{tabular}

Fuente: Elaboración propia (2006). 


\subsection{Valores}

Los valores se constituyen en creencias que orientan las acciones individuales y grupales de una sociedad, pues como lo señalan Secord y Backman (2000), son ideas compartidas por los miembros de un grupo o cultura, lo cual complementa Papua (2002) al considerarlos como marcos de referencia que sirven de guía para la evaluación de la experiencia y de la conducta.

Por otra parte, Cárdenas (2002), ve los valores como una creencia consistente y motivación que activa y jerarquiza las decisiones que subyacen a la conducta del ser humano, mientras Bruna (2002), considera que el sistema social genera sus propios valores, por medio de superestructuras que funcionan como entes socializantes entre los que se encuentran el medio familiar, educativo, religioso, político y laboral.

Con los enfoques de estos autores y en base al marco de la responsabilidad social empresarial, se identificó la presencia de la RSE en los valores de las franquicias mediante la percepción de confianza, pulsando las opiniones sobre promoción de los valores asumidos por estas empresas y su orientación hacia el mejoramiento de la calidad de vida de su fuerza laboral, así como el trabajar por el

\section{Gráfico 3}

\section{Presencia de RSE en los valores}

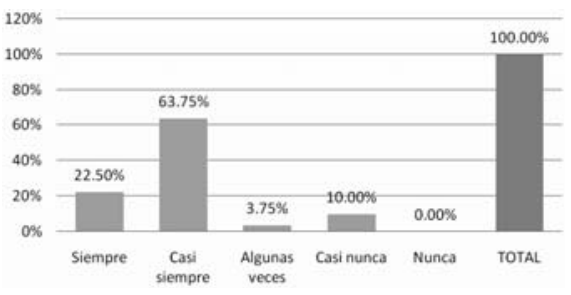

Fuente: Elaboración propia (2006).

bien social, obteniendo los resultados mostrados en la Tabla 3 y Gráfico 3.

Como puede observarse a partir del indicador "Percepción de la confianza", las respuestas de los sujetos alcanzaron un $86,25 \%$ entre las alternativas "siempre" y "casi siempre" con énfasis en ésta última, sobre la promoción de los valores al personal y el conocimiento de la orientación a mejorar la calidad de vida de la fuerza laboral en las franquicias farmacéuticas dónde trabajan, así como al bienestar social, lo cual parte de creencias e ideas compartidas que conforman un marco de referencia, indicativo de una conducta socialmente responsable en ambas organizaciones.

Se destaca en los resultados que por otra parte el $13,75 \%$ de los gerentes entrevistados difirió en la opinión, considerando que sólo algunas veces o casi nunca hay percepción de la confianza en

\section{Tabla 3}

\section{Presencia de RSE en los valores}

\begin{tabular}{lccccccc}
\hline $\begin{array}{l}\text { Indicador: } \\
\text { Percepción }\end{array}$ & Categorías & Siempre & Casi siempre & $\begin{array}{c}\text { Algunas } \\
\text { veces }\end{array}$ & Casi nunca & $\begin{array}{c}\text { Nunca } \\
\text { de la }\end{array}$ & $\begin{array}{c}N^{\circ} \text { de } \\
\text { Sujetos }\end{array}$ \\
\cline { 2 - 7 } confianza & F. Absoluta & 4.5 & 12.75 & 0.75 & 2 & 0 & 20 \\
& $\%$ & $22.5 \%$ & $63,75 \%$ & $3.75 \%$ & 10 & - & $100 \%$ \\
\hline
\end{tabular}

Fuente: Elaboración propia (2006). 
promoción y conocimiento de los valores orientados en el ámbito de la RSE.

\subsection{Políticas}

De acuerdo con Stoner (2003), las políticas son guías para la toma de decisiones, las cuales establecen límites y canalizan el pensamiento de los empleados para que sea compatible con los objetivos de la empresa. Por otra parte, Carneiro (2004) señala que las responsabilidades primarias inmediatas de la empresa se vinculan con los integrantes de la propia organización (accionistas, directivos y trabajadores).

En ese orden de ideas, se revisó si las empresas utilizaban como política mejorar el conocimiento de la fuerza laboral relativo a especificaciones de los productos o servicios que ofrecen y las garantías, mediante la Percepción sobre especificaciones técnicas para la garantía producto/servicio dentro del ámbito de la RSE, obteniéndose los resultados mostrados en la Tabla 4 y Gráfico 4.

Como indica la Tabla 4, a partir del indicador "Percepción sobre conocimiento de especificaciones técnicas para garantía producto/servicio" se encontró que el $96,25 \%$ de las respuestas correspondió a las frecuencias "siempre" y "casi

\section{Gráfico 4 Presencia de RSE en las políticas}

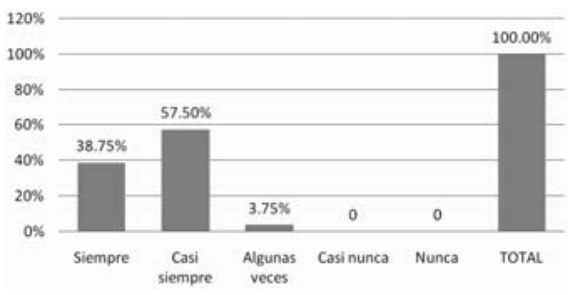

Fuente: Elaboración propia (2006).

siempre", con el mayor peso en esta última $(57,50 \%)$.

Coincidiendo con lo señalado por Carneiro (2004), estos resultados mostraron una tendencia positiva hacia la formación de los empleados de estas organizaciones en responsabilidades primarias inmediatas en las cuales se vinculan sus integrantes, orientándose a garantizar el producto o servicio brindado, trabajando por el bien social dentro del ámbito de la RSE.

\subsection{Normas}

Según Melinkoff (2000), normas son guías o propósitos que conducen las operaciones en la dirección deseada, a través de contenidos específicos y correctamente aplicables. Por su parte,

\section{Tabla 4}

Presencia de RSE en las políticas

\begin{tabular}{lccccccc}
\hline $\begin{array}{l}\text { Indicador: Percepción } \\
\text { sobre conocimiento de }\end{array}$ & Categorías & Siempre & $\begin{array}{c}\text { Casi } \\
\text { siempre }\end{array}$ & $\begin{array}{c}\text { A } \\
\text { veces }\end{array}$ & $\begin{array}{c}\text { Casi } \\
\text { nunca }\end{array}$ & \begin{tabular}{c} 
Nunca \\
especificaciones técnicas para de \\
\cline { 2 - 8 } garantía producto/servicio
\end{tabular} \\
\cline { 2 - 8 } & F. Absoluta & 7,8 & 11,5 & 0,7 & 0 & 0 & 20 \\
\hline
\end{tabular}

Fuente: Elaboración propia (2006). 
Munch y García (2000), consideran que son mandatos precisos, los cuales determinan la disposición o actividad que deberá seguir o evitar la organización para el logro de los objetivos. De acuerdo con tales planteamientos, se buscó determinar si ambas franquicias contaban con la formulación de normas dirigidas a lograr objetivos de trabajar por el bien social y mejorar la calidad de vida de la fuerza laboral, dentro del ámbito de la RSE. Los resultados se presentan a continuación en la Tabla 5 y Gráfico 5.

En la Tabla 5 se puede observar como para el indicador "formulación de normas", el $85 \%$ de las respuestas consideró que siempre o casi siempre estas organizaciones tenían formuladas las normas que orientaban sus operaciones en la dirección deseada y eran difundidas entre los miembros, orientadas al bien social y con mandatos precisos en relación con los aspectos de mejora de calidad de vida que atañen al personal de las mismas. En el Gráfico se aprecian los resultados expresados en porcentajes.

Se encontró que el $15 \%$ restante presentó discrepancias, pues señalaban que solamente a veces las normas estaban orientadas al bien social y dirigidas a mejorar la calidad de vida de los trabajadores.

\section{Gráfico 5 Presencia de RSE en las normas}

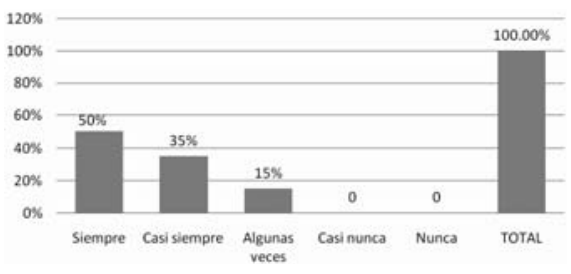

Fuente: Elaboración propia (2006).

Al consolidar los resultados, se tiene la información resumida en la Tabla 6 y presentada en valores porcentuales en el Gráfico 6.

Puede apreciarse en la Tabla 6 como los elementos estratégicos concentran el $90 \%$ de respuestas positivas tomando en cuenta las alternativas "siempre" y "casi siempre", destacándose como los gerentes de estas franquicias perciben la presencia de la responsabilidad social empresarial en la misión, visión, políticas y normas, manifestado mediante un comportamiento responsable del personal y directivos de las franquicias farmacéuticas estudiadas.

El 10\% restante se ubica en las alternativas "algunas veces" y "casi Nunca", apreciándose la opinión contraria de una minoría, mostrada en su percepción sobre la presencia de la RSE en los elementos estratégicos considerados.

\section{Tabla 5}

Presencia de RSE en las normas

\begin{tabular}{lccccccc}
\hline & Categorías & Siempre & $\begin{array}{c}\text { Casi } \\
\text { siempre }\end{array}$ & $\begin{array}{c}\text { Algunas } \\
\text { veces }\end{array}$ & $\begin{array}{c}\text { Casi } \\
\text { nunca }\end{array}$ & $\begin{array}{c}\text { Nunca } \\
\text { Indicador: }\end{array}$ & $\begin{array}{c}N^{\circ} \text { de } \\
\text { Sormulación }\end{array}$ \\
\cline { 2 - 8 } de Normas & F. Absoluta & 10 & 7 & 3 & 0 & 0 & 20 \\
\hline & $\%$ & $50 \%$ & $35 \%$ & $15 \%$ & - & - & $100 \%$ \\
\hline
\end{tabular}

Fuente: Elaboración propia (2006). 


\section{Tabla 6}

\section{Elementos estratégicos de las empresas}

\begin{tabular}{|c|c|c|c|c|c|c|c|}
\hline \multirow{2}{*}{\multicolumn{2}{|c|}{ Elemento }} & \multicolumn{6}{|c|}{ Alternativa } \\
\hline & & \multirow{2}{*}{$\begin{array}{c}\text { Siempre } \\
11,75\end{array}$} & \multirow{2}{*}{$\begin{array}{c}\begin{array}{c}\text { Casi } \\
\text { siempre }\end{array} \\
5,5\end{array}$} & \multirow{2}{*}{$\begin{array}{c}\begin{array}{c}\text { Algunas } \\
\text { veces }\end{array} \\
2\end{array}$} & \multirow{2}{*}{$\begin{array}{c}\text { Casi nunca } \\
0,75\end{array}$} & \multirow{2}{*}{$\begin{array}{c}\text { Nunca } \\
0\end{array}$} & $\begin{array}{l}\text { No Sujetos } \\
\text { Informantes }\end{array}$ \\
\hline Misión & & & & & & & \\
\hline Visión & & 11 & 8,25 & 0,75 & 0 & 0 & \\
\hline Valores & & 4,5 & 12,75 & 0,75 & 2 & 0 & \\
\hline Políticas & & 7,75 & 11,5 & 0,75 & 0 & 0 & \\
\hline Normas & & 10 & 7 & 3 & 0 & 0 & \\
\hline \multirow{2}{*}{ Resultados } & $\bar{x}$ & 9 & 9 & 1,45 & 0,55 & 0 & 20 \\
\hline & $\%$ & $45 \%$ & $45 \%$ & $7,25 \%$ & $2,75 \%$ & & $100 \%$ \\
\hline
\end{tabular}

Fuente: Elaboración propia (2006).

\section{Gráfico 6}

\section{Presencia de la RSE en los elementos estratégicos de las franquicias}

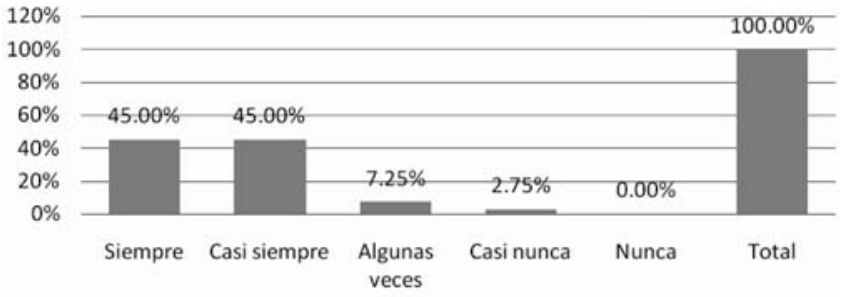

- CATEGORÍAS

Fuente: Elaboración propia (2006).

"Casi siempre" reúne la mayor frecuencia de respuestas, indicando que los Elementos Estratégicos de las franquicias farmacéuticas están orientados en gran medida hacia la Responsabilidad Social Empresarial.

\section{Conclusiones}

La tendencia hacia la responsabilidad social empresarial, identificada en los elementos estratégicos propios de las franquicias farmacéuticas consideradas, está presente en la misión, visión, políticas y normas de las mismas, y se manifiesta mediante un comportamiento responsable del personal y directivos de estas empresas.

Esto lleva a la conclusión de que en ellas se logra el compromiso del personal con la RSE, estableciéndose una comunicación clara del concepto hacia el entorno social y consistencia del compromiso de los integrantes de las franquicias farmacéuticas en el municipio Maracaibo, con sus relacionados, proveedores y clientes. 
Por otra parte en dichas empresas, se tiene claro lo que desean llegar a ser en el mercado, en el sentido de posicionarse en los lugares de vanguardia proyectando metas sobre sus actividades. Las personas entrevistadas reconocen la existencia de valores orientados hacia la RSE en las franquicias farmacéuticas estudiadas, existiendo la percepción de que hay confianza, por parte de los clientes y proveedores en particular así como de la colectividad marabina en general, en los productos/servicios que brindan a su entorno social.

Asimismo, se considera que las políticas asumidas por las franquicias contribuyen al comportamiento socialmente responsable, dentro del ámbito de la RSE, por cuanto están dirigidas al conocimiento por parte de los empleados de las especificaciones técnicas y a garantizar el producto/servicio ofrecido al entorno, así como trabajar responsablemente atendiendo a los proveedores, clientes y comunidad en general.

Adicionalmente, los encuestados reconocen la existencia de normas en las franquicias farmacéuticas, formuladas para orientar sus operaciones en la dirección deseada, orientadas al bien social y difundidas entre los miembros con mandatos precisos en relación a los aspectos de mejora de calidad de vida que atañen al personal de las mismas.

En síntesis, las franquicias farmacéuticas estudiadas en el municipio Maracaibo del Estado Zulia, presentan características positivas en la orientación hacia la responsabilidad social mediante un comportamiento socialmente responsable que incorpora el concepto, según las percepciones de los gerentes medios de estas dos empresas.

Los resultados llevan a reconocer la presencia de la responsabilidad social empresarial en los elementos estratégicos de ambas empresas, pero resalta la necesidad de profundizar en su asimilación y afianzamiento, con el fin de fortalecer las acciones para cumplir la obligación ética y moral asumida por cada una de estas empresas y poder medirse el impacto que genera el cumplimiento de dicha obligación, de acuerdo a los objetivos escogidos dentro del marco de la RSE y en los cuales estén comprometidos, en función de mejorar calidad de vida y satisfacción de demandas de orden social a nivel interno y externo de dichas organizaciones.

Pero además, mantener y mejorar el comportamiento responsable con la sociedad luce como una estrategia que representa un factor de competitividad de primer orden, pues las partes involucradas o grupos de interés (clientes, proveedores, competidores, comunidad, empleados, gobierno) tienen expectativas cada vez más exigentes; no hacerlo traería como consecuencia pérdida de mercado, desconfianza y rechazo, aspectos totalmente contrarios para lograr el éxito y la permanencia en el tiempo.

\section{Referencias bibliográficas}

Álvarez, E. (2007 a). El paradigma de la gestión empresarial responsable. Otro Mundo es Posible. Revista Iberoamericana de Sostenibilidad. (on line) julio 2007, año 3 Número 25. http://www.otromundoesposible.net. Consulta: 22-08-2007. 
Álvarez, E. (2007 b). La Responsabilidad Social Corporativa (RSC) y el mercado. Otro Mundo es Posible. Revista lberoamericana de Sostenibilidad. (on line) mayo 2007, año 3 Número 23. http://www.otromundoesposible.net. Consulta: 22-08-2007.

Asociación Venezolana de Franquicias (2005). www.franquiciasonline.com. Consulta: 18-01-2006.

Bull, B. (2004). ¿Responsabilidad social de las empresas: ¿Una solución para el desarrollo en América Latina? Revista Venezolana de Gerencia, dic.2004, Vol. 9 No. 28, p.565-584. ISSN 13159984.

Bruna, H. (2002). Como incrementar la Calidad-Productividad en su Empresa. México: Editorial McGraw-Hill Interamericana. México.

González, I.A. y otros (2003). Responsabilidad Social Empresarial. Análisis, comparaciones y propuestas sobre el comportamiento del "ciudadano empresa". Normativa y realidades. Una visión integral incluyendo Argentina. IDEA: Buenos Aires.

Cárdenas, L. (2002). La educación en Valores. Un reto compartido. Caracas: Taller Universo.

Carneiro, Manuel (2004). La Responsabilidad Social Corporativa Interna: La Nueva frontera de los Recursos Humanos. Madrid: ESIC Editorial.

Chávez, Nilda (2000). Introducción a la Investigación Educativa. Maracaibo: Editorial Ars. Gráfica, S.A.

Consejo Empresarial Mundial para el Desarrollo Sostenible (2002). http://sustainable-finance.org/web/publications/business-case-spanish.pdf. Consulta: 24-01-2006

Hernández, Fernández y Baptista (2003). Principios de la Metodología de la
Investigación. México: Editorial McGraw-Hill

Comisión de Comunidades Europeas. (2001). Libro Verde COM (2001) 366 final.http://www.ecodes.org/documentosecores/Libro_verde_RSC.pdf. Consulta: 24-01-2006

Melinkoff, Ramón (2000). Los Procesos Administrativos. Caracas: Editorial $\mathrm{Pa}$ napo

Méndez, Charo (2003). Responsabilidad social de empresarios y empresas en Venezuela durante el siglo XX. Caracas: Impresión Gráfica ACEA.

Morrisey, George (2002). Pensamiento Estratégico. México: Editorial Prentice Hall.

Munch y García (2002). Fundamentos de Administración. Madrid: Editorial Trillas.

Papua (2002). La Cultura Organizacional y el Liderazgo. Funciones de la cultura en las empresas. Madrid: Plaza y Janes Editores.

Parra, Javier (2000). Guía de Muestreo Maracaibo: Colección XLV Aniversario. FCES.

Secord y Backman (2000). Psicología en el comportamiento en las organizaciones. $3^{\mathrm{a}}$ Edición. Barcelona España: Ediciones Díaz de Santos.

Stoner, James (2003). Administración. Tr. Arturo Córdova. $5^{\mathrm{a}}$ Edición. México: Editorial Prentice Hall Hispanoamericana, S.A.

Thompson, Andrés (2000). Programa de Ética para la América Latina y Caribe de la Fundación W.K. Kellogg. http://www.iarse.org/newsletter/n_006/columnista_invitado.htm. Consulta: 7-02-2006

Vives, Antonio et al. (2005). Responsabilidad Social de la Empresa en las Pymes de Latinoamérica. Washington, DC: Banco Interamericano de Desarrollo. 